\title{
Optimized protocol for imaging cleared neural tissues using light microscopy
}

Authors: Yoh Isogai ${ }^{1,4}$, Douglas Richardson ${ }^{2}$, Catherine Dulac ${ }^{1}$, Joseph Bergan $^{3}$

Affiliations:

1. Howard Hughes Medical Institute, Department of Molecular and Cellular Biology, Harvard University

2. Harvard Center for Biological Imaging, Harvard University

3. Department of Psychological and Brain Sciences, University of Massachusetts, Amherst

4. Sainsbury Wellcome Centre for Neural Circuits and Behaviour, University College London

Running title:

Optimized protocol for imaging cleared tissues

Keywords:

lightsheet, confocal, CLARITY, OptiView, tissue clearing, whole brain imaging 


\begin{abstract}
Understanding physical and chemical processes at an organismal scale is a fundamental goal in biology. While science is adept at explaining biological phenomena at both molecular and cellular levels, understanding how these processes translate to organismal functions remains a challenging problem. This issue is particularly significant for the nervous system where cell signaling and synaptic activities function in the context of broad neural networks. Recent progress in tissue clearing technologies lessens the barriers that previously prevented the study of large tissue samples while maintaining molecular and cellular resolution. While these new methods open vast opportunities and exciting new questions, the logistics of analyzing cellular processes in intact tissue have to be carefully considered. In this protocol, we outline a procedure to rapidly image intact brain tissue up to thousands of cubic millimeters. This experimental pipeline involves three steps: tissue clearing, tissue imaging, and data analysis. In an attempt to streamline the process for researchers entering this field, we address important considerations for each of these stages and describe an integrated solution to image intact biological tissues. Hopefully, this optimized protocol will lower the barrier of implementing highresolution tissue imaging and facilitate the investigations of mesoscale questions at molecular and cellular resolution.
\end{abstract}




\section{Introduction}

Mapping the structure and connectivity of neural circuits is essential to understand brain function in both healthy and diseased states. A powerful set of tools already exists to characterize neural circuits, processes, synapses, and proteins at multiple spatial resolutions. For example: classic retrograde and anterograde tracing techniques efficiently map the broad connections between brain regions [1-3], viral strategies allow entire neural circuits to be traced from genetically defined neural populations [4,5], and histological techniques such as antibody staining and in situ hybridization allow the precise localization of individual biomolecules $[6,7]$. Central to each of these techniques is the need to analyze the location, shape, and connectivity of individually labeled neurons, synapses, or proteins. In the past, processing histological brain tissue typically required sectioning samples into thin ( 10-100 um) sections to allow visualization of deep structures. The process of sectioning tissue can be time consuming, difficult to automate, prone to tissue imperfections (tearing and folding), and destroys many essential long-range connections. Recent advances in tissue clearing techniques, however, make mapping neural circuits within large volumes of intact tissue both feasible and practical [817].

Techniques for making biological samples transparent date back more than a century $[18,19]$, and the potential for these strategies to image large volumes of intact tissue at high resolution is finally being realized (Figure 1, 2 and 3). Each tissue clearing protocol has both drawbacks and benefits, most of which have been discussed previously [20,21]. Instead, our goal here is to: 1) Outline a cost-effective and reliable protocol in sufficient detail to rapidly produce high-quality tissue samples; 2) Indicate factors within this protocol that are particularly sensitive and provide guidance on the selection of these parameters; 3 ) Describe the process of preparing tissue for imaging; 4) Compare and contrast microscopy strategies for imaging large volumes of brain tissue; 5) Discuss important considerations for the analysis of the potentially massive datasets generated by this approach.

The procedures outlined below are intended as a reasonable entry point for imaging large volumes of intact tissue; however, some refining of these procedures will undoubtedly be required to optimize results with each unique preparation. Important parameters that may require tuning are indicated throughout the protocol.

\section{Materials}

\subsection{Sample considerations}

Fluorophores of choice (see Note 1 and 2)

\subsection{Electrophoresis equipment}

Parts list for buffer re-circulating system for CLARITY electrophoretic clearing (see Note 3)

\begin{tabular}{lll}
\hline Vendor & Item description & Catalog No. \\
\hline
\end{tabular}




\begin{tabular}{|c|c|c|}
\hline Grey Beard Pumps & $\begin{array}{l}\text { The Niagara with } 120 \text {-Volt Transformer - } \\
\text { Designed for Water }\end{array}$ & 316 \\
\hline US Plastic & 3" Opening 5 Gallon Carboy & A00357 \\
\hline KORAD & $\begin{array}{l}\text { Precision Variable Adjustable 60V 5A } \\
\text { DC Linear Power Supply }\end{array}$ & KA6005D \\
\hline McMaster-Carr & Water Filter with light-blocking blue bowl & $4448 K 35$ \\
\hline McMaster-Carr & Replacement Filter Cartridge & $4422 K 61$ \\
\hline McMaster-Carr & $\begin{array}{l}\text { Patch Cord - Banana Plug to Alligator } \\
\text { Clip (12") }\end{array}$ & $6927 \mathrm{~K} 42$ \\
\hline McMaster-Carr & 1/4" Tubing - 5 feet & $2085 T 5$ \\
\hline McMaster-Carr & Single Barbed Tube Fitting & $5463 K 247$ \\
\hline McMaster-Carr & $\begin{array}{l}\text { Single barbed tube fitting ( } 7 / 16 " \text { hex for } \\
1 / 4 \text { tubing) }\end{array}$ & $5463 K 245$ \\
\hline McMaster-Carr & $\begin{array}{l}\text { Durable Nylon Tight-Seal Barbed Tube } \\
\text { Fitting }\end{array}$ & $5463 K 247$ \\
\hline
\end{tabular}

\subsection{Refractometer (Milwaukee MA871)}

\subsection{Chemical reagents}

Hydrogel monomer solution: $4 \%$ acrylamide, 0.0,25 \% bisacrylamide, $0.25 \%$ VA-044 initiator (Wako), 1x PBS, 4 \% paraformaldehyde

Clearing solution: $200 \mathrm{mM}$ boric acid, pH8.5, 4 \% SDS [8]

Superglue

Imaging medium: FocusClear (CelExplorer Labs) or OptiView (172 mM sodium diatrizoate, 815 $\mathrm{mM}$ meglumine diatrizoate, $0.5 \mathrm{mM}$ EDTA, $0.1 \%$ Tween-20, $\mathrm{pH}$ 8, refractive index adjusted to 1.46 using a refractometer, filtered through $0.22 \mu \mathrm{m}$ pore filter) (see Note 4 )

\subsection{Microscopy Equipment}

Most imaging systems were not designed to image samples centimeters in size; however, with careful consideration of the working distance of available objectives, sample mounting technique, and refractive index of the final clearing solution a number of homebuilt and commercially available confocal, multi-photon, and lightsheet microscopes can be adapted for this task.

\subsubsection{Objectives}

The objectives available will determine the maximum depth in the sample can be imaged, what lateral and axial resolutions can be obtained, and which microscopy techniques are best suited to the experiment.

\subsubsection{Working Distance}

In a properly cleared, highly transparent tissue, imaging depth is most often limited by objective working distance. Routinely, only low numerical aperture (NA) objectives (less than 0.5) have a working distance greater than $2 \mathrm{~mm}$. Unfortunately, if the NA is too low, 3D imaging is not 
worthwhile due to the poor axial resolution. Currently, researchers must choose between one of three options.

1. Recommended: a number of long working distance (above $5 \mathrm{~mm}$ ), high NA (more than 0.9) objectives are now commercially available and can be used in conjunction with confocal, multi-photon and lightsheet microscopes.

2. Alternative \#1: a low/moderate NA (less than 0.6), long working distance objective can be used in a lightsheet configuration where the thinness of the lightsheet, and not the imaging objective, determines the axial resolution $[26,14,17]$.

3. Alternative \#2: an objective with a modest NA and long working distance (at least $3 \mathrm{~mm}$ ) can be used in conjunction with a chamber that places the sample between two coverslips [8]. Here, approximately half of the sample is imaged from each side and the two 3D volumes are merged using image processing software.

\subsubsection{Refractive Index}

Most moderate to high NA, long working distance objectives are corrected to the refractive index (RI) of water (1.33). Many of the modern clearing solutions, including OptiView, have an RI close to 1.46 . This refractive index mismatch (1.33 versus 1.46$)$ will increase aberration, reducing imaging depth and resolution in all dimensions. Again, three options exist:

1. Recommended: use of objectives that are specifically matched to a refractive index of 1.45 , or objectives with correction collars that can be tuned over this range. These are now commercially available.

2. Alternative \#1: Use a moderate to low NA water immersion objective. Although, not perfect, the refractive index of water, 1.33 , is a closer match than air. Additionally, the lower NA will be less susceptible to aberration and the objective will have a longer working distance.

3. Alternative \#2: The introduction of adaptive optics to some imaging platforms can be used to correct for aberrations and improve imaging depth and resolution [27].

\subsubsection{Imaging Modality}

Two commercially available modalities exist that can be adapted to imaging large, cleared tissue samples: point-scanning confocal (see Note 6), and lightsheet microscopy.

\subsubsection{Point-Scanning Confocal Microscopy}

Most commercially available confocal microscopes can be used (Figure 1), however, the following modifications are highly recommended.

1. Upright microscope (inverted microscopes are poorly suited to image large samples).

2. Large $Z$ (axial) objective travel range

3. Adjustable height stage

4. Fully automated $(x, y)$ mechanical stage

5. Long working distance, RI-matched objective (see Section 2.4.1.2)

6. Full compliment of yellow and red laser lines (561 nm, 594 nm, $633 \mathrm{~nm}$, tunable IR) 
7. 80/20 transmission/reflection dichroic mirror (allows imaging of reflected light, see Section 3.2 below)

\subsubsection{Lightsheet Microscopy}

Lightsheet imaging of large, cleared tissues has mainly been performed using two designs, both of which have now been commercialized:

1. Ultramicroscopy [9] uses a setup similar to a dissecting microscope. The sample is placed in the base of the microscope containing optics that produce a thin lightsheet that can be focused through the sample. A long working distance, low NA air objective or moderate NA dipping objective capable of capturing a large field of view sits above the sample. Here the entire sample can be imaged in a single field of view, or zoom optics and a mechanical stage can be used to reduce the field of view and tile and stitch together a final image. Fluorescence captured by the imaging objective is projected onto a camera.

2. Selective plane illumination microscopy [28] suspends the sample in front of a standard compound microscope objective between two additional excitation objectives that project a lightsheet into the sample. In this system, the sample is raster or 'tile' scanned in front of the imaging objective as it is too large to fit in a single field of view. The fluorescence captured by the imaging objective is delivered to a camera. By removing the need for laser scanning, an entire $Z$ plane can be captured in as little at 10 milliseconds. This means an entire brain can be imaged in a few hours or less, depending on the field of view of the microscope (Figure 2 and 3).

Recommended components for a lightsheet microscope:

1. Cylindrical lens to form the lightsheet

2. Dual side excitation light paths

3. Dual view light path and two sCMOS cameras for simultaneous two channel fast read out

4. Adjustable mirror to ensure lightsheet is positioned at focal plane of imaging objective

5. Resonance scanning mirror to provide a pivot scan to reduce line artifacts (see Section 2.4.4 below; [29]

6. Laser blocking filter for all but one excitation laser (allows imaging of scattered light, see Section 3.2 below)

2.5 High performance computer system with large bandwidth and storage (see Note 5 for recommended specifications)

\subsection{Image processing software}

Several open source and commercial software packages exist to facilitate processing large imaging datasets. The following freeware/open source options are recommended:

1. FIJI / ImageJ / BioFormats are useful to convert proprietary file formats to .tif, downsample data, and stitch tiles [30,31]. Additionally, a large and growing number of plugins are available 
that enable a wide range of procedures (image filtering, cell counting, co-localization, and many more).

2. Vaa3d [32] allows fast and detailed 3D visualization of image stacks. While currently more limited in analysis capabilities, the array of plugins available for Vaa3d is rapidly growing (Figure 3).

3. Commercial software suites such as Arivis Vision4D (Arivis AG) and Imaris (Bitplane) are also capable of handling TB-sized data sets, provide 3D rendering, and allow a diverse range of data analysis tools.

\section{Methods}

\subsection{Electrophoretic clearing of mouse brains}

Caution: Hydrogel solution contains formaldehyde and acrylamide and is highly toxic. Perform perfusion strictly under a ventilation hood with proper protective gear and dispose of waste chemicals under institutional guidelines.

1. Perfuse animal with $50 \mathrm{ml}$ PBS followed by $25 \mathrm{ml}$ hydrogel monomer solution and transfer the brain tissue to a sealable tube containing $\sim 25 \mathrm{ml}$ cold hydrogel solution [8].

2. Flush excess oxygen from the tissue-containing tube with nitrogen gas.

3. Keep brains at $4^{\circ} \mathrm{C}$ (with aluminum foil to shield them from light) for 2 days, inverting 1-2 times on the second day.

4. After 24 hours (this may be extended), replace oxygen with nitrogen a second time.

Because oxygen inhibits acrylamide polymerization, it is important to remove as much oxygen from the tissue containing tube as possible before initiating acrylamide polymerization (step

5). Alternating rounds of exerting a vacuum on the uncapped tissue-containing tube and replacing the removed gases with pure nitrogen can easily achieve this. Repeating this step 3 times evenly spaced over 15 minutes is sufficient.

5. Bring a water bath to $38^{\circ} \mathrm{C}$ and move the tube containing the brain directly from ice into this water bath (see Note 7).

6. Carefully remove the brain from the hydrogel, and gently roll the brain across a Kimwipe to take off any remaining hydrogel. (see Note 8).

7. Wash the brain in PBST overnight at room temperature while rocking

8. Wash the brain in clearing solution overnight at room temperature while rocking (See Note 9)

9. Place brain into an electrophoresis chamber, tighten the cap, and cover with aluminum foil to prevent exposure to ambient light.

10. Turn on the pump and allow clearing solution to flow for several minutes before turning on the electrophoresis power supply. 
11. Adjust the voltage to achieve an initial current of $\sim 0.5 \mathrm{~A}$. If using one of the designs that can be found at http://clarityresourcecenter.org/, this will likely require a voltage of $\sim 30 \mathrm{~V}$. It is important to monitor the chamber temperature to ensure that the temperature does not rise above $45^{\circ} \mathrm{C}$. The current source should be immediately shut down if the temperature exceeds $45^{\circ} \mathrm{C}$ to prevent damage to the tissue and reduction of fluorescence.

12. The duration required to clear a tissue sample depends primarily on the size of the tissue sample and the length of time the tissue has been allowed to passively clear. Tissue samples less than $2 \mathrm{~mm}$ are likely to be cleared after $\sim 24$ hours of electrophoresis. Larger samples may require up to 4 days. This can be done all at once or broken into parts to maintain appropriate temperature and voltage. The temperature should always stay between 37 and $45^{\circ} \mathrm{C}$.

13. Remove the brain and wash overnight at room temperature in PBST while rocking.

14. Place brains in $200 \mathrm{~mL}$ of OptiView solution for 3-5 days prior to imaging. Prior to imaging, move brain into a fresh $50 \mathrm{~mL}$ of OptiView solution for 1 day. Closely monitor $\mathrm{pH}(8.0)$ and refractive index (1.46) of this solution, as it will slowly be diluted with PBST if reused. For optimal results replace solution when refractive index begins to drop. Gently invert several times once a day to ensure thorough mixing (see note 4.9).

\subsection{Imaging cleared brains}

\subsubsection{Sample Mounting}

Determining the best way to mount samples requires consideration of both the imaging system and objective to be used. Coverslip-corrected objectives require a coverslip to be placed between the sample and the objective. Ensure the coverslip is the correct thickness (usually $\# 1.5,0.17 \mathrm{~mm}$ ) and that the coverslip rests perfectly flat. It is absolutely essential to remove all air bubbles from the mounted sample, as it is impossible to image through air bubbles regardless of the specific imaging strategy (see below). If the working distance of the objective is insufficient to cover the entire sample, sandwich the sample between two coverslips and image from both sides. A suggested dual coverslip chamber design is presented in Chung et al., 2013. A more convenient option is to use a dipping objective on an upright microscope. In this configuration the sample can be glued to a petri dish, which is filled with the final clearing solution, assuming the objective has the required working distance. Finally, certain lightsheet designs require the sample to be suspended in a fluid filled chamber. One successful strategy is to glue a small magnet to the base of the tissue away from any regions of interest, and use this magnet to reversibly mount the tissue.

Whether using confocal or lightsheet imaging, it may be helpful to acquire an additional data channel of auto-fluorescence or reflected light. This is best done with a long wavelength (>600 $\mathrm{nm}$ ) laser as it has the best ability to penetrate through the tissue. This dataset can serve multiple purposes including: 1) image registration and 2) subtraction of the tissue autofluorescence signal from fluorescently labeled channels.

\subsubsection{Imaging with an upright point scanning confocal microscope (see Note 10)}

1. Superglue brain to bottom of a high-walled 60-100 $\mathrm{mm}$ Petri dish

2. Fill petri dish with OptiView solution until brain is submerged 
3. Lower stage of upright microscope if required to allow for clearance of long working distance objective and large sample

4. Submerge objective (if dipping) and acquire tiled Z-stacks over area of interest with 5-10\% overlap between tiles and an optical zoom of 1-1.3 (to reduce tiling edge artifacts in final image).

\subsubsection{Imaging by lightsheet microscopy}

1. Orient mounted brains such that any areas of interest are $>1 \mathrm{~mm}$ from the attachment site.

2. Fill the imaging chamber with OptiView, and lower the brain into the microscope chamber.

3. Allow the tissue sample to rest in the imaging chamber for several hours prior to imaging (optional, see Note 9). This allows time to equalize any refractive index differences that may have developed during the mounting procedure, air bubbles at the top of the imaging chamber to disperse, and temperature differences to normalize. During this step, it is important to cover the imaging chamber to prevent evaporation of the imaging medium.

4. Acquire tiled z-stacks of the desired regions with at least $5 \%$ overlap between tiled views. $5 \%$ overlap is important to ensure the images can be accurately stitched together into a single dataset. Often, it is helpful to image large tissue samples from multiple angles, as distinct regions of any tissue sample will be imaged most clearly when the light path distance is minimized.

5. After imaging, remove brain and place in PBST at $4^{\circ} \mathrm{C}$ for storage.

\subsection{Data merging / Image processing}

If the sample is larger than a few $\mathrm{mm}$ in any direction, it is likely that multiple image stacks will need to be stitched together, and a variety of tools exist to make this process easier, more accurate, and efficient. Two options stand out as particularly effective for large datasets.

1. The grid/Collection stitching feature in FIJI [30] is easy to use and suitable for small datasets. As the program is memory intensive, large datasets must be processed in virtual stack mode, which increases processing time. An advantage of this method is that the output transformation can be applied to images of any size and/or color that share the same reference frame.

2. Terastitcher - Built as a standalone program, as well as a plugin for FIJI and Vaa3D. Terastitcher provides a fast and accurate means to assemble multiple tiled image stacks into a large 3D volume dataset and allows non-rigid stitching [33]. Terastitcher is more efficient with memory usage and suitable for larger datasets.

\subsection{Reducing Artifacts}

The most common artifacts seen during imaging are dark lateral (lightsheet) or axial (confocal) stripes. These are most often caused by the introduction of air bubbles into the sample or hydrogel, the accumulation of debris on the sample surface, or poorly cleared tissue regions. 
Lightsheet microscopes are more susceptible to casting shadows behind areas of strong staining due to the high absorption of excitation light in these regions. To reduce these artifacts:

1. Only handle the sample in a clean environment. Do not allow towels or blotting paper that will deposit lint to come in contact with the sample.

2. Avoid puncturing/damaging the hydrogel or sample with sharp forceps or other dissection tools.

3. Keep samples fully submerged in imaging medium at all times and transfer tissue samples (that are not enclosed by a coverslip) to the imaging chamber as quickly as possible to reduce the introduction of air bubbles.

4. Prior to filling the sample holder/imaging chamber ensure it is completely dry and no aqueous solutions are present that could alter the RI of the imaging solution.

5. In a lightsheet microscope, illuminating the sample from both the left and right sides relative to the imaging objective can reduce artifacts provided both lightsheets penetrate to the current field of view.

6. The introduction of a resonance scanning mirror that "pivots" the lightsheet allowing it to enter the sample from multiple angles can greatly reduce stripe artifacts [29]. However, care should be taken with this step as pivot scanning may introduce additional noise if the refractive index of the medium is not properly calibrated for the objectives.

7. Many image processing software packages have a line or stripe removal option. This often relies on identifying stripes in a Fourier transformation of the image and removing that data from the Fourier transform. Caution: this is not compatible with intensity-based analyses as required data may be removed.

\subsection{Troubleshooting}

1. Inconsistent clearing:

Perhaps the most common cause for inconsistent clearing is that the tissue sample is highly myelinated. White matter is more resistant to the clearing process, and often requires longer durations of active or passive clearing. If the white matter remains opaque, one should extend the clearing time until better results are obtained. Extending the duration of passive clearing at $37^{\circ} \mathrm{C}$ can also be effective. If extending the clearing procedure is not successful, it will likely be necessary to reduce the tissue size.

2. Cleared brains have brownish tint and/or burnt spots:

One common problem of the electrophoretic step is that brains obtain a yellow or brown color. Some yellowing should be expected, however, excessive voltage, duration, or heat during clearing can potentiate this issue. It is recommended to lower the voltage and current and to monitor the temperature carefully. Another approach is to clear tissue in multiple short periods of time. For example, alternating hours of active and passive clearing. Tissue that comes into close, or direct, contact with electrodes may show burnt spots. Make sure that the 
electrophoresis chamber has enough room so that the brain does not come in direct contact with the electrodes.

3. Low fluorescence compared to freshly fixed tissues:

There will be some fluorescence loss from the clearing procedure. However, further fluorescence loss can be minimized by avoiding excessive heat in the electrophoresis chamber and by avoiding over-clearing.

4. Yellowing of the clearing solution:

The clearing solution can turn slightly yellow over time, but this does not seem to affect the clearing step or fluorescence intensity. Carefully follow the initial conditions as outlined in Section 3.1. Clearing solution should be renewed when, or preferably before, there is a change in electrical resistance -as evidenced by a noticeable difference in the current to voltage relationship or an increase in heat production.

5. Image stitching failure; tiles do not perfectly register:

When imaging a large tissue sample at single cell resolution, it is extremely important to keep the sample steady throughout the acquisition. It is important to acquire multiple tiled stacks such that the order of tiles minimizes sample movement. A failure to keep the sample steady will result in altered three-dimensional geometry of the sample, and it can cause errors in stitching. Also, consider the working distance of the objectives. Frequently, the objective working distance will determine the maximum depth that can be imaged. Attempting to image deeper than appropriate for the given objective will result in distorted images that are inaccurate and will be difficult to stitch together.

6. Blurry images:

Blurry images often result from a mismatch in refractive index of the sample and the imaging medium. We recommend: 1) measuring the precise refractive index of each batch of imaging medium; 2) Maintain the samples in the same solution for at least 2 days prior to imaging while periodically adjusting the refractive index to the desired level; 3) Transfer samples with the same solution for imaging without the introduction of air bubbles; 4) Ensure that the imaging chamber is completely dry before filling with imaging medium; 5) Adjust the refractive index of the imaging medium to optimize the balance between tissue clarity, refractive index mismatch with the objectives, and distortion in the lightsheet angles.

\section{Notes}

4.1 Maximizing the strength of fluorescence and the transparency of each tissue sample are primary concerns. Longer wavelength fluorophores (e.g., tdTomato) should be chosen whenever possible as the excitation wavelengths required for excitation can penetrate deeper, even in cleared tissue, and will likely provide more consistent results. The following protocol has been successfully used to image tissues expressing mCherry, GFP, YFP, mCitrine, and tdTomato transgenically, as well as tissues expressing these fluorescent proteins from viral 
constructs. Shorter wavelength fluorescent proteins (e.g., BFP) have been imaged in deep tissues with only limited success.

4.2 Secondary labeling of tissue, for example by antibody staining, has been successful. However, one should currently expect secondary labeling procedures to take several weeks (if not months) when performed on large tissue samples. This is an active area of research and it is likely the antibody staining procedures will become more efficient in the near future. As before, choosing a fluorophore that is shifted towards the longer wavelengths and also provides a strong signal is essential.

4.3 The success of clarifying biological tissues relies on generating a tissue sample with a uniform refractive index. The CLARITY procedure [8] achieves this by removing lipids from the tissue sample with electrophoresis. A low-cost buffer recirculation system for this electrophoretic step can be constructed using the parts listed in Materials. This setup can be constructed for under $\$ 1000$ and the electrophoretic step can be performed optimally at $37^{\circ} \mathrm{C}$.

4.4 The selection of imaging medium is a critical step since optimized tissue clearing requires the refractive index of tissue and medium to be carefully matched $[22,23]$. However, not all refractive index matched solutions are equivalent. For example, organic solvents such as methyl salicylate and BABB clear well [24], but contain toxic compounds that can also damage microscope components. Many organic solvents also shrink tissue, possibly compromising the underlying structure, and displace water molecules that are essential for maintaining fluorescence from many fluorophores, including nearly all fluorescent proteins. Glycerol based media are cheap and allow precise refractive index matching, but in our experience, the achieved transparency tends to be unstable, making long imaging experiments and tissue storage difficult. FocusClear, a commercially available solution [22,25], works well for CLARITY cleared brains [8]. However, the cost of proprietary reagents may be prohibitively expensive for some experiments.

The lack of a low-cost imaging medium that maintains the original structure, provides suitable transparency of samples, and does not quench fluorescence signals motivated us to develop a new transparentizing reagent. This reagent, OptiView, consists of defined chemical components that can be titrated to optimize the refractive index for different samples. For imaging of CLARITY-treated mouse brains, we found that a refractive index of 1.46 is optimal.

4.5 When imaging datasets that require the acquisition of many $Z$ stacks (tiles), data sets can quickly become prohibitively large. Depending on the number of tiles required and number of channels imaged, file sizes may exceed several terabytes in size. Therefore, planning how to store and analyze the data prior to data collection is essential. In the case of lightsheet microscopy, data is acquired extremely rapidly requiring a computer with fast write speeds. Although solid state hard drives are likely not necessary, a RAID array comprised of multiple 
7200 rpm hard disk drives is a must. In addition, if different workstations are being used for acquisition and storage, a high-speed $10 \mathrm{Gbit}$ network connection is recommended. It is also recommended to have 100-200 GB of RAM for both acquisition (to buffer images if there is a slowdown in data writing) and processing as some data analysis steps can require large amounts of memory. It is possible to reduce the need for RAM with most image analysis software suites by using virtual image stacks; however, in many cases this will noticeably slow image processing. Access to cluster computing may also be advantageous as many image processing steps can be performed in parallel.

4.6. Spinning disk confocal microscopy is not well suited for imaging thick samples as the pinhole size and spacing of most disks are optimized for high NA objectives with short working distances. The largest drawback to point-scanning confocal is its slow speed. Depending on the chosen field of view, and lateral and axial resolutions, imaging of an entire brain would likely take days. Therefore, point scanning confocal is ideal for generating high-resolution 3D images of smaller regions and not generally useful for imaging an entire organ. Green/red/IR lasers are recommended as blue and near-UV lasers have trouble penetrating deep into tissue even when cleared.

4.7. For best passive clearing results, add a stir bar to the bath to cause the brain to rock, and cover the tube containing the brain with aluminum foil.

4.8. We use the same clearing solution as indicated in Chung et al. 2013. During the electrophoretic process a dark residue that reduces the imaging quality may be deposited on the tissue surface. One solution to this problem is to remove most, but not all of the excess hydrogel prior to the electrophoretic step. Once the tissue has been processed through the electrophoretic step, the remaining hydrogel can be removed along with the unwanted residue.

Additionally, passive clearing for several days at $37^{\circ} \mathrm{C}$ can reduce the required duration of electrophoretic tissue clearing and minimize damage from the prolonged heating during electrophoresis. Passive clearing by extending the incubation with clearing solution also maintains the highest level of fluorescence and can therefore be used to increase the final signal-to-noise ratio of genetically encoded fluorescent proteins.

4.9 OptiView can transparentize a $1 \mathrm{~mm}$ slice of fixed mouse brain without electrophoretic clearing within 1 2 days. It can be pushed to clear 2 3 $\mathrm{mm}$ tissues taking about a week but white matter will remain cloudy. In all cases, better transparency is achieved with longer incubation times. If possible, it is helpful to incubate the tissue sample for as long as possible prior to imaging. 
4.10. In highly transparent, well-cleared tissue, multi-photon excitation is often not needed as visible wavelength lasers using a single photon process can often penetrate the entire working distance of the objective. As a single photon excitation event is more probable than a multiphoton event, this can result in a higher signal-to-noise ratio and the ability to scan faster [34]. However, multi-photon offers two advantages over single photon: reduced out-of-focal-planebleaching and increased penetration for excitation of blue/green emitting dyes [34] (see Section 2.5.2.1).

\section{Figure legends}

Figure 1. Three dimensional reconstruction (1.2 $\mathrm{mm} \times 1.2 \mathrm{~mm} \times 0.4 \mathrm{~mm}$ ) of vomeronasal sensory neuron axons terminating in the accessory olfactory bulb. Transgenically expressed tdTomato in specific vomeronasal receptor neurons is imaged using a Zeiss LSM710 confocal microscope with a 10x objective immersed in OptiView. Optical sections were 3D rendered using Imaris.

Figure 2. Three-dimensional reconstruction of fluorescently labeled neurons in the olfactory bulb. A) A $1.25 \mathrm{~mm} \times 1.25 \mathrm{~mm} \times 3.5 \mathrm{~mm}$ region of a CLARITY treated mouse olfactory bulb was placed in OptiView and imaged with a Zeiss Lightsheet Z.1 microscope. TdTomato was expressed in mitral and tufted cells by crossing a Tbet:Cre line [35] with the Ai9 tdTomato reporter line [36]. Initial image processing was done using FIJI, and the volume image was rendered using the maximum intensity projection rendering algorithm in Vaa3D. B) An individual imaging plane acquired at a depth of $1 \mathrm{~mm}$ (arrow panel $A$ ) with lightsheet microscopy.

Figure 3. Three-dimensional reconstruction of cell bodies and processes of fluorescently labeled neurons in the olfactory bulb. A) A $600 \mu \mathrm{m} \times 600 \mu \mathrm{m} \times 150 \mu \mathrm{m}$ region of mouse olfactory bulb (same conditions as in figure 1) was imaged with a Zeiss Lightsheet Z.1 microscope. Initial image processing was done using $\mathrm{FIJI}$, and the volume image was rendered using the maximum intensity projection rendering algorithm in Vaa3D. Axons, dendrites, and potential synaptic contacts are easily observed with this imaging strategy.

\section{Acknowledgement}

We would like to thank the Howard Hughes Medical Institute and the National Institute of Health (NIH/NIDCD RO1 5R01DC013087-02; F32 DC10089) for funding. We also thank the Harvard Center for Biological Imaging for providing infrastructure and support. 


\section{References}

1. Petrovich GD, Canteras NS, Swanson LW (2001) Combinatorial amygdalar inputs to hippocampal domains and hypothalamic behavior systems. Brain research Brain research reviews $38(1-2): 247-289$

2. Swanson LW (2000) Cerebral hemisphere regulation of motivated behavior. Brain research $886(1-2): 113-164$

3. Graybiel AM, Ragsdale CW, Jr. (1979) Fiber connections of the basal ganglia. Progress in brain research 51:237-283

4. Watabe-Uchida M, Zhu L, Ogawa SK, Vamanrao A, Uchida N (2012) Whole-brain mapping of direct inputs to midbrain dopamine neurons. Neuron 74 (5):858-873.

doi:10.1016/j.neuron.2012.03.017

5. Wickersham IR, Lyon DC, Barnard RJ, Mori T, Finke S, Conzelmann KK, Young JA, Callaway EM (2007) Monosynaptic restriction of transsynaptic tracing from single, genetically targeted neurons. Neuron 53 (5):639-647. doi:10.1016/j.neuron.2007.01.033

6. Lein ES, Hawrylycz MJ, Ao N, Ayres M, Bensinger A, Bernard A, Boe AF, Boguski MS, Brockway KS, Byrnes EJ, Chen L, Chen L, Chen TM, Chin MC, Chong J, Crook BE, Czaplinska A, Dang CN, Datta S, Dee NR, Desaki AL, Desta T, Diep E, Dolbeare TA, Donelan MJ, Dong HW, Dougherty JG, Duncan BJ, Ebbert AJ, Eichele G, Estin LK, Faber C, Facer BA, Fields R, Fischer SR, Fliss TP, Frensley C, Gates SN, Glattfelder KJ, Halverson KR, Hart MR, Hohmann JG, Howell MP, Jeung DP, Johnson RA, Karr PT, Kawal R, Kidney JM, Knapik RH, Kuan CL, Lake JH, Laramee AR, Larsen KD, Lau C, Lemon TA, Liang AJ, Liu Y, Luong LT, Michaels J, Morgan JJ, Morgan RJ, Mortrud MT, Mosqueda NF, Ng LL, Ng R, Orta GJ, Overly CC, Pak TH, Parry SE, Pathak SD, Pearson OC, Puchalski RB, Riley ZL, Rockett HR, Rowland SA, Royall JJ, Ruiz MJ, Sarno NR, Schaffnit K, Shapovalova NV, Sivisay T, Slaughterbeck CR, Smith SC, Smith KA, Smith BI, Sodt AJ, Stewart NN, Stumpf KR, Sunkin SM, Sutram M, Tam A, Teemer CD, Thaller C, Thompson CL, Varnam LR, Visel A, Whitlock RM, Wohnoutka PE, Wolkey CK, Wong VY, Wood M, Yaylaoglu MB, Young RC, Youngstrom BL, Yuan XF, Zhang B, Zwingman TA, Jones AR (2007) Genome-wide atlas of gene expression in the adult mouse brain. Nature 445 (7124):168-176. doi:10.1038/nature05453

7. Oh SW, Harris JA, Ng L, Winslow B, Cain N, Mihalas S, Wang Q, Lau C, Kuan L, Henry AM, Mortrud MT, Ouellette B, Nguyen TN, Sorensen SA, Slaughterbeck CR, Wakeman W, Li Y, Feng D, Ho A, Nicholas E, Hirokawa KE, Bohn P, Joines KM, Peng H, Hawrylycz MJ, Phillips JW, Hohmann JG, Wohnoutka P, Gerfen CR, Koch C, Bernard A, Dang C, Jones AR, Zeng H (2014) A mesoscale connectome of the mouse brain. Nature 508 (7495):207-214. doi:10.1038/nature13186

8. Chung K, Wallace J, Kim SY, Kalyanasundaram S, Andalman AS, Davidson TJ, Mirzabekov JJ, Zalocusky KA, Mattis J, Denisin AK, Pak S, Bernstein H, Ramakrishnan C, Grosenick L, Gradinaru V, Deisseroth K (2013) Structural and molecular interrogation of intact biological systems. Nature 497 (7449):332-337. doi:10.1038/nature12107

9. Dodt HU, Leischner U, Schierloh A, Jahrling N, Mauch CP, Deininger K, Deussing JM, Eder M, Zieglgansberger W, Becker K (2007) Ultramicroscopy: three-dimensional visualization of neuronal networks in the whole mouse brain. Nature methods 4 (4):331-336.

doi:10.1038/nmeth1036

10. Erturk A, Becker K, Jahrling N, Mauch CP, Hojer CD, Egen JG, Hellal F, Bradke F, Sheng M, Dodt HU (2012) Three-dimensional imaging of solvent-cleared organs using 3DISCO. Nat Protoc 7 (11):1983-1995. doi:10.1038/nprot.2012.119

11. Hama H, Kurokawa $H$, Kawano H, Ando R, Shimogori T, Noda H, Fukami K, SakaueSawano A, Miyawaki A (2011) Scale: a chemical approach for fluorescence imaging and reconstruction of transparent mouse brain. Nat Neurosci 14 (11):1481-1488.

doi:10.1038/nn.2928 
12. Ke MT, Fujimoto S, Imai T (2013) SeeDB: a simple and morphology-preserving optical clearing agent for neuronal circuit reconstruction. Nat Neurosci 16 (8):1154-1161.

doi:10.1038/nn.3447

13. Kuwajima T, Sitko AA, Bhansali P, Jurgens C, Guido W, Mason C (2013) ClearT: a detergent- and solvent-free clearing method for neuronal and non-neuronal tissue. Development 140 (6):1364-1368. doi:10.1242/dev.091844

14. Susaki EA, Tainaka K, Perrin D, Kishino F, Tawara T, Watanabe TM, Yokoyama C, Onoe H, Eguchi M, Yamaguchi S, Abe T, Kiyonari H, Shimizu Y, Miyawaki A, Yokota H, Ueda HR (2014) Whole-brain imaging with single-cell resolution using chemical cocktails and computational analysis. Cell 157 (3):726-739. doi:10.1016/j.cell.2014.03.042

15. Tainaka KSIK, S.I.; Suyama, T.Q.; Susaki, E.A.; Perrin, D.; Ukai-Tadenuma, M.; Ukai, H.; Ueda, H.R. (2014) Whole-body imaging with single-cell resolution by tissue decolorization. Cell $159(4): 911-924$

16. Yang B, Treweek JB, Kulkarni RP, Deverman BE, Chen CK, Lubeck E, Shah S, Cai L, Gradinaru V (2014) Single-cell phenotyping within transparent intact tissue through whole-body clearing. Cell 158 (4):945-958. doi:10.1016/j.cell.2014.07.017

17. Renier NW, Z.; Simon, D.J.; Yang, J.; Ariel, P.; Tessier-Lavigne, M. (2014) iDISCO: A Simple, Rapid Method to Immunolabel Large Tissue Samples for Volume Imaging. Cell 159

(4):896-910

18. Schultze O (1897) Über Herstellung and Conservirung durchsichtigen Embryonen zum Stadium der Skeletbildung. In: Verhandlungen der Anatomischen Gesellschaft, vol 13.

Anatomischer Anzeiger, pp 3-5

19. Spalteholz W (1914) Über das Durchsichtigmachen von menschlichen und tierischen

Präparaten. S. Hierzel, Leipzig

20. Chung K, Deisseroth K (2013) CLARITY for mapping the nervous system. Nature methods 10 (6):508-513. doi:10.1038/nmeth.2481

21. Kim SY, Chung K, Deisseroth K (2013) Light microscopy mapping of connections in the intact brain. Trends in cognitive sciences 17 (12):596-599. doi:10.1016/j.tics.2013.10.005 22. Zhu D, Larin KV, Luo Q, Tuchin VV (2013) Recent progress in tissue optical clearing. Laser \& photonics reviews 7 (5):732-757. doi:10.1002/lpor.201200056

23. Efimova OI, Anokhin KV (2009) Enhancement of optical transmission capacity of isolated structures in the brain of mature mice. Bulletin of experimental biology and medicine 147 (1):3-6 24. Oldham M, Sakhalkar H, Oliver T, Allan Johnson G, Dewhirst M (2008) Optical clearing of unsectioned specimens for three-dimensional imaging via optical transmission and emission tomography. Journal of biomedical optics 13 (2):021113. doi:10.1117/1.2907968 25. Genina EA, Bashkatov AN, Tuchin VV (2010) Tissue optical immersion clearing. Expert review of medical devices 7 (6):825-842. doi:10.1586/erd.10.50

26. Tomer R, Ye L, Hsueh B, Deisseroth K (2014) Advanced CLARITY for rapid and highresolution imaging of intact tissues. Nat Protoc 9 (7):1682-1697. doi:10.1038/nprot.2014.123 27. Wang K, Milkie DE, Saxena A, Engerer P, Misgeld T, Bronner ME, Mumm J, Betzig E (2014) Rapid adaptive optical recovery of optimal resolution over large volumes. Nat Methods 11 (6):625-628. doi:10.1038/nmeth.2925

28. Huisken J, Swoger J, Del Bene F, Wittbrodt J, Stelzer EH (2004) Optical sectioning deep inside live embryos by selective plane illumination microscopy. Science 305 (5686):1007-1009. doi:10.1126/science.1100035

29. Huisken J, Stainier DY (2007) Even fluorescence excitation by multidirectional selective plane illumination microscopy (mSPIM). Opt Lett 32 (17):2608-2610 30. Preibisch S, Saalfeld S, Tomancak P (2009) Globally optimal stitching of tiled 3D microscopic image acquisitions. Bioinformatics 25 (11):1463-1465.

doi:10.1093/bioinformatics/btp184 
31. Schindelin J, Arganda-Carreras I, Frise E, Kaynig V, Longair M, Pietzsch T, Preibisch S, Rueden C, Saalfeld S, Schmid B, Tinevez JY, White DJ, Hartenstein V, Eliceiri K, Tomancak P, Cardona A (2012) Fiji: an open-source platform for biological-image analysis. Nat Methods 9 (7):676-682. doi:10.1038/nmeth.2019

32. Peng H, Bria A, Zhou Z, lannello G, Long F (2014) Extensible visualization and analysis for multidimensional images using Vaa3D. Nature protocols 9 (1):193-208.

doi:10.1038/nprot.2014.011

33. Bria A, lannello G (2012) TeraStitcher - a tool for fast automatic 3D-stitching of teravoxelsized microscopy images. BMC Bioinformatics 13:316. doi:10.1186/1471-2105-13-316 34. Denk W, Strickler JH, Webb WW (1990) Two-photon laser scanning fluorescence microscopy. Science 248 (4951):73-76

35. Haddad R, Lanjuin A, Madisen L, Zeng H, Murthy VN, Uchida N (2013) Olfactory cortical neurons read out a relative time code in the olfactory bulb. Nat Neurosci 16 (7):949-957. doi:10.1038/nn.3407

36. Madisen L, Zwingman TA, Sunkin SM, Oh SW, Zariwala HA, Gu H, Ng LL, Palmiter RD, Hawrylycz MJ, Jones AR, Lein ES, Zeng H (2010) A robust and high-throughput Cre reporting and characterization system for the whole mouse brain. Nat Neurosci 13 (1):133-140.

doi:10.1038/nn.2467 
Figure 1

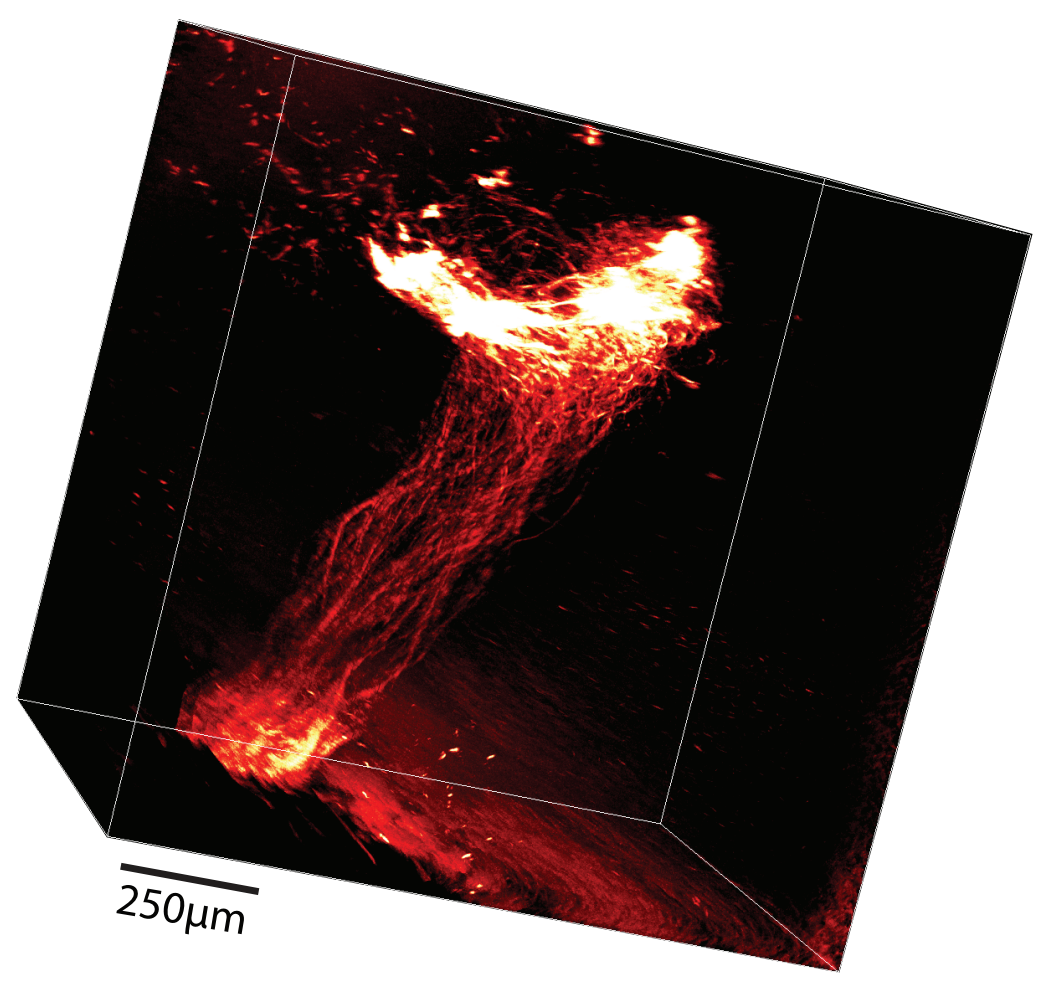


Figure 2
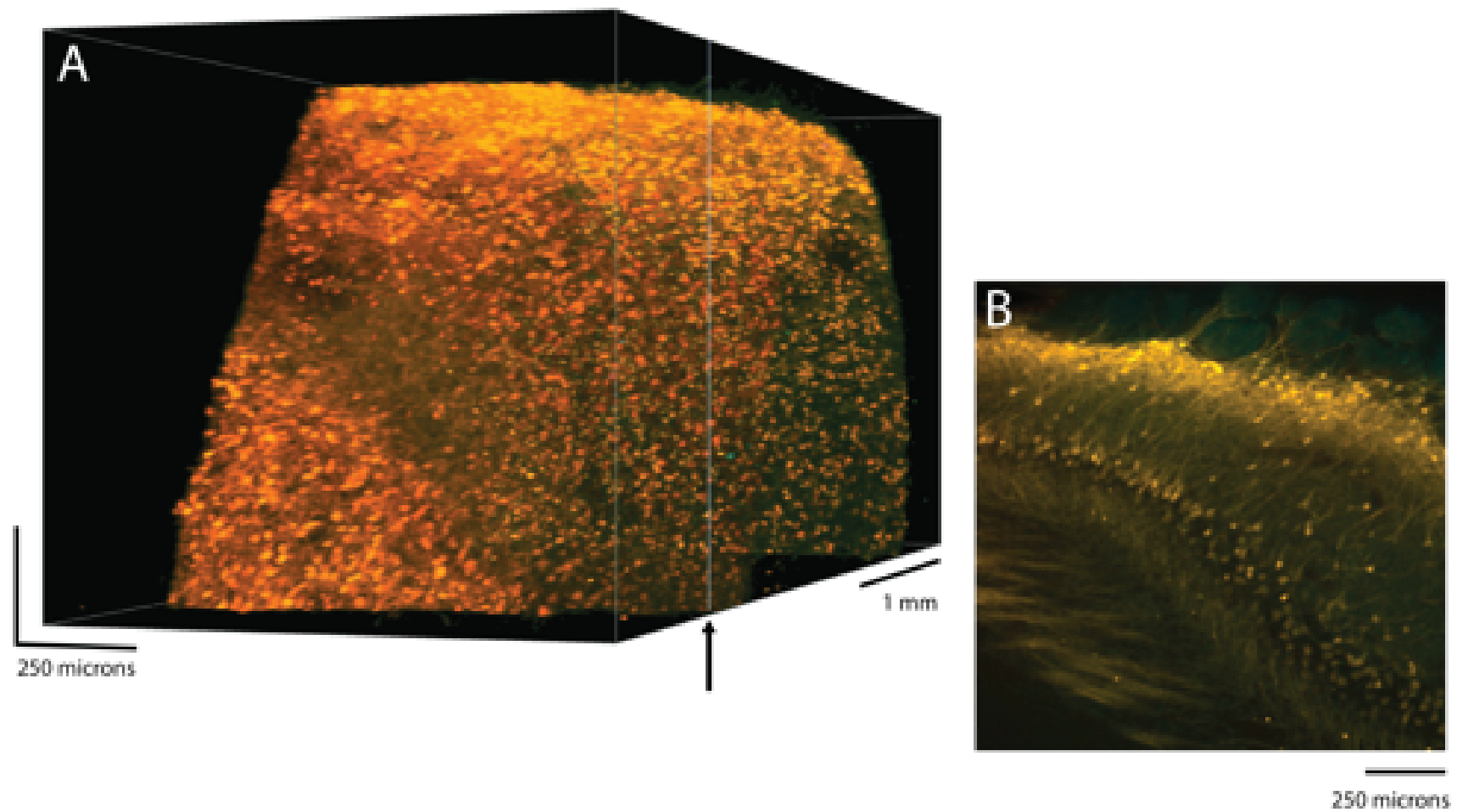
Figure 3

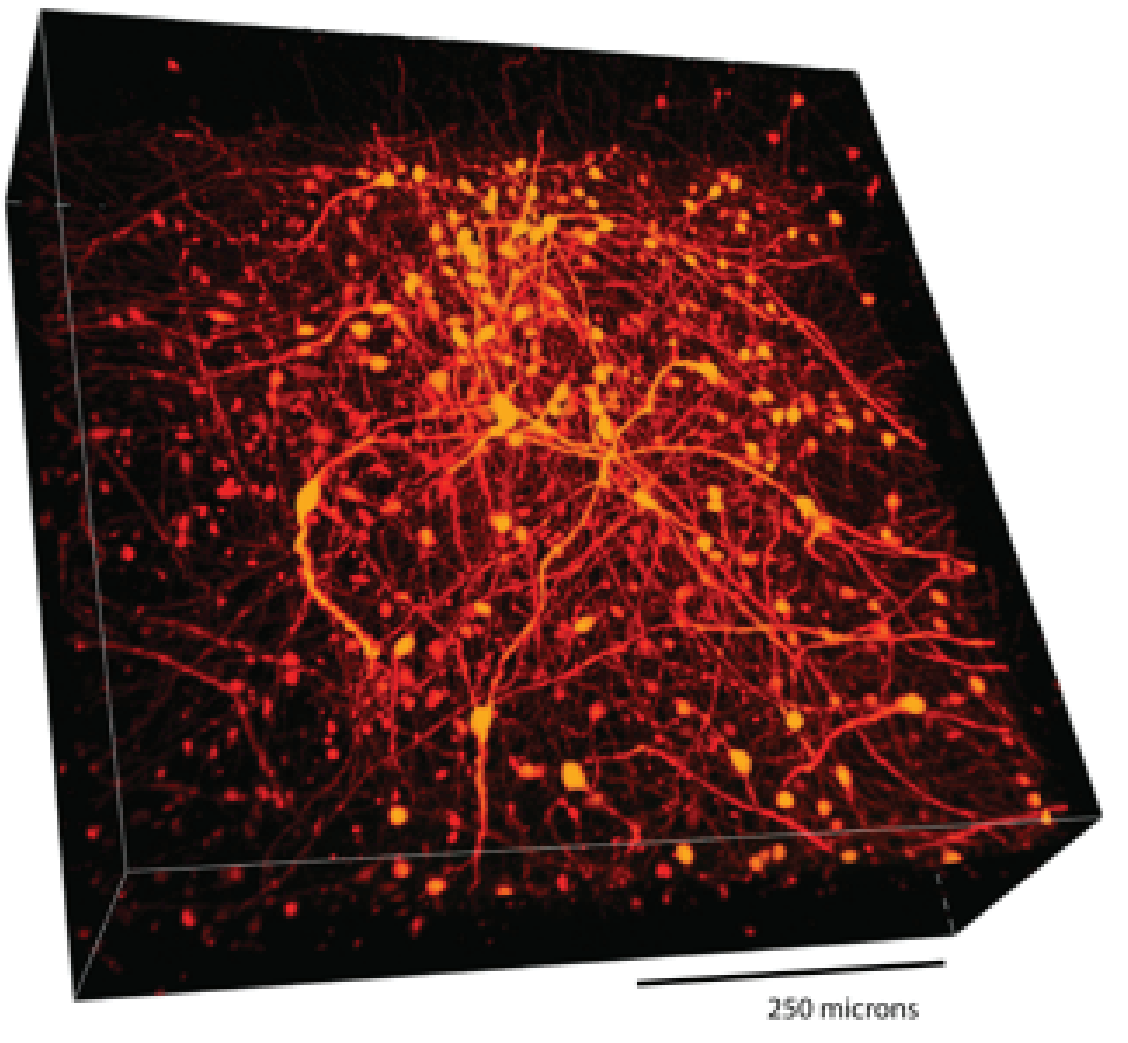

